\title{
Factors associated with high consumption of soft drinks among Australian secondary-school students
}

\author{
Maree Scully ${ }^{1}$, Belinda Morley ${ }^{1}, *$, Philippa Niven ${ }^{1}$, David Crawford ${ }^{2}$, lain S Pratt ${ }^{3}$ and \\ Melanie Wakefield ${ }^{1}$ for the NaSSDA Study Team \\ ${ }^{1}$ Centre for Behavioural Research in Cancer, Cancer Council Victoria, 615 St Kilda Road, Melbourne, VIC 3004, \\ Australia: ${ }^{2}$ Institute for Physical Activity and Nutrition (IPAN), Deakin University, Melbourne, Australia: ${ }^{3}$ Cancer \\ Council Western Australia, Perth, Australia
}

Submitted 25 July 2016: Final revision received 13 December 2016: Accepted 13 January 2017: First published online 27 February 2017

\begin{abstract}
Objective: To examine demographic and behavioural correlates of high consumption of soft drinks (non-alcoholic sugar-sweetened carbonated drinks excluding energy drinks) among Australian adolescents and to explore the associations between high consumption and soft drink perceptions and accessibility.

Design: Cross-sectional self-completion survey and height and weight measurements. Setting: Australian secondary schools.

Subjects: Students aged 12-17 years participating in the 2012-13 National Secondary Students' Diet and Activity (NaSSDA) survey ( $n$ 7835).

Results: Overall, $14 \%$ of students reported consuming four or more cups ( $\geq 1$ litres) of soft drinks each week ('high soft drink consumers'). Demographic factors associated with high soft drink consumption were being male and having at least \$AU 40 in weekly spending money. Behavioural factors associated with high soft drink consumption were low fruit intake, consuming energy drinks on a weekly basis, eating fast foods at least once weekly, eating snack foods $\geq 14$ times/week, watching television for $>2 \mathrm{~h} / \mathrm{d}$ and sleeping for $<8 \mathrm{~h} /$ school night. Students who perceived soft drinks to be usually available in their home, convenient to buy and good value for money were more likely to be high soft drink consumers, as were students who reported usually buying these drinks when making a beverage purchase from the school canteen/vending machine.

Conclusions: High soft drink consumption clusters with other unhealthy lifestyle behaviours among Australian secondary-school students. Interventions focused on reducing the availability of soft drinks (e.g. increased taxes, restricting their sale in schools) as well as improved education on their harms are needed to lower adolescents' soft drink intake.
\end{abstract}

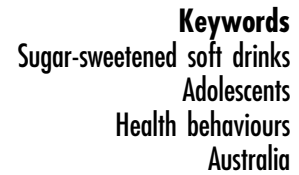

Sugar-sweetened soft drinks ('soft drinks') are energydense and offer little nutritional value. Consumption of these carbonated beverages is associated with increased energy intake as individuals do not adequately adjust their overall food intake to compensate for the extra energy provided by these drinks ${ }^{(1)}$. Sugar-sweetened beverages (SSB), which include soft drinks as well as energy drinks, fruit drinks, sports drinks and cordial, have been linked to weight gain and obesity in both adults and children ${ }^{(2-4)}$. The acidity of soft drinks also likely contributes to dental erosion and caries in children ${ }^{(5,6)}$.

In Australia, adolescents are among the highest consumers of soft drinks ${ }^{(7)}$. Cross-sectional data from the 2007 Australian Children's Nutrition and Physical Activity Survey found that $38 \%$ of males and $28 \%$ of females aged $14-16$ years reported consuming soft drinks on the day of the survey, with a mean intake of $591 \mathrm{~g}$ and $507 \mathrm{~g}$, respectively (compared with $600 \mathrm{~g} / 470 \mathrm{~g}$ for cordial and $473 \mathrm{~g} / 311 \mathrm{~g}$ for fruit drinks) ${ }^{(8)}$. Although a decline in the proportion of Australian children consuming soft drinks has been observed since the 1995 National Nutrition Survey ${ }^{(9)}$, current prevalence levels are still of concern given the negative health impacts associated with these beverages. Consequently, interventions aimed at reducing intake of soft drinks among young Australians are needed.

An understanding of factors related to adolescents' soft drink consumption is important to enable interventions to be targeted where they are likely to have most impact. While soft drinks belong within the broader category of SSB and are often studied at this combined level, prior 
research among middle- and high-school students in Texas suggests that dietary and activity correlates of SSB may vary by type of $\operatorname{SSB}^{(10)}$. For example, consumption of non-carbonated flavoured and sports beverages was found to be positively associated with several healthy behaviours including fruit and vegetable intake and physical activity, whereas soft drink consumption (referred to as 'regular soda' in the USA) showed either no association or a negative association ${ }^{(10)}$. Such patterns are unsurprising given that sport drinks are typically marketed in a way that aligns them with exercise and optimal athletic performance. However, these possible differences between specific types of SSB indicate there may be value in considering soft drinks as their own distinct category.

Globally, there have been few large national studies that have investigated factors associated with soft drink consumption among adolescent populations. Using data from 11029 US high-school students surveyed as part of the 2010 National Youth and Physical Activity and Nutrition Study, researchers explored cross-sectional associations between consumption of regular soda ( $\geq 1$ time/d) and a number of characteristics including age, sex, weight status, availability of school vending machines, physical activity, television viewing and frequency of eating at fast-food restaurants ${ }^{(11)}$. They found that drinking regular soda on a daily basis was positively associated with being male, less physically active, watching higher amounts of television and eating at fastfood restaurants more regularly. In a similar US school-based study (2009 Youth Risk Behavior Survey; $n$ 16188) that examined a more comprehensive list of dietary and other behavioural factors, daily sugar-sweetened soda intake was found to be positively associated with male sex, inadequate sleep, spending more time in sedentary behaviours, being less physically active and cigarette smoking, but inversely associated with low intake of vegetables and trying to lose weight ${ }^{(12)}$. A Norwegian study of 9th and 10th graders ( $n$ 2870) which took an ecological approach and assessed personal and environmental factors, in addition to demographic factors, found that taste preferences, accessibility of soft drinks at home, modelling of the behaviour by family/ friends and positive attitudes towards soft drinks, along with being male and dieting, were the strongest determinants of adolescents' soft drink consumption ${ }^{(13)}$. Clifton et al. utilised data from 4834 Australian children aged 2-16 years who participated in the 2007 Australian National Children's Nutrition and Physical Activity Survey to look at how soft drink consumption related to socio-economic position (SEP), physical activity and television viewing ${ }^{(8)}$. Their analyses found that lower SEP and higher television viewing hours were associated with higher intake of soft drinks.

The aim of the present study was to build on this relatively small body of research by examining associations between high consumption of soft drinks and selected demographic characteristics, weight status and health behaviours among Australian adolescents. Given the influence of personal and environmental factors on behaviour $^{(14)}$, we also aimed to explore whether adolescents' perceptions of the availability, convenience and value for money of soft drinks, and the purchasing of soft drinks at school, is associated with their consumption.

\section{Method}

\section{Design and procedure}

Data were obtained from students participating in the 2012-13 National Secondary Students' Diet and Activity (NaSSDA) survey. A nationally representative sample of students from Years 8 to 11 (aged 12-17 years) was surveyed from schools across Australia. The sampling procedure used a stratified two-stage probability design, with schools randomly selected at the first stage of sampling and classes selected within schools at the second stage. Within each State and Territory, schools were stratified by the three education sectors (government, Catholic and independent) and randomly selected from each sector to ensure that the distribution of schools by sector within each State or Territory was reflected in the sample.

\section{Measures}

\section{Soft drink consumption}

Students' consumption of soft drinks (i.e. all non-alcoholic sugar-sweetened carbonated drinks excluding energy drinks) was assessed by asking: 'Thinking about just soft drinks such as Coke and lemonade, how much soft drinks do you usually drink?' ( 1 cup $=250 \mathrm{ml}$, one can of soft drink $=1 \frac{1}{2}$ cups). Students were specifically instructed not to include diet soft drinks. Response options included: 'I don't drink soft drinks'; 'less than one cup a week'; 'about 1-3 cups a week'; 'about 4-6 cups a week'; 'about 1-2 cups a day'; 'about 3-4 cups a day'; and '5 cups or more a day'. Students who indicated they consumed four or more cups ( $\geq 1$ litres) per week were classified as 'high soft drink consumers'.

\section{Demographic characteristics}

All students reported their sex, school year level and home postcode. A measure of SEP was determined according to the Socio-Economic Index for Areas Index of Relative Socio-Economic Disadvantage, based on the student's home postcode ${ }^{(15)}$. This index ranks areas on a continuum of disadvantage (from most disadvantaged to least disadvantaged), taking into consideration characteristics that may enhance or reduce socio-economic conditions of the area, such as income, education, occupation and housing. Using the national deciles to create quintiles, students were categorised into the following SEP groups: low (first and second quintiles); medium (third and fourth quintiles); and high (fifth quintile). Postcode of residence was also used to classify the geographic location of students as metropolitan or rural/regional according to the Australian Statistical Geography Standard Remoteness Structure ${ }^{(16)}$. 
Finally, students reported the amount of money they have available to spend on themselves (e.g. from pocket money, part-time job) during a typical week, with responses ranging from 'none' to '\$AU 100 per week or more'.

\section{Weight status}

Measurements of students' height and weight, taken in accordance with standardised protocols by trained researchers $^{(17)}$, were used to calculate students' BMI $\left(=\right.$ weight $/$ height $\left.^{2}\right)$. Students were categorised as overweight or obese based on internationally recognised cut-offs developed for children and adolescents ${ }^{(18)}$.

\section{Health behaviours}

Items from a short dietary questionnaire developed by the NSW Centre for Public Health Nutrition were used to assess students' core and non-core food consumption habits ${ }^{(19)}$. Using the provided response categories, students reported the number of servings of vegetables (excluding potatoes, hot chips and fried potatoes) and fruit (excluding fruit juice) they usually consume each day, the number of cups of fruit juice and non-alcoholic energy drinks they usually drink and how often they have meals or snacks (such as burgers, pizza, chicken or chips) from fast-food or takeaway-food places. Four items ('ice cream, icy poles or ice blocks', 'potato crisps/chips or other salty snacks', 'confectionery' and 'sweet foods') were used to measure students' frequency of consuming snack foods (a detailed description of how this variable was computed has been reported elsewhere ${ }^{(20)}$ ).

The $60 \mathrm{~min}$ moderate-to-vigorous physical activity screening measure was used to assess physical activity, which has been shown to be reliable, valid and correlate well with accelerometer data ${ }^{(21)}$. Television viewing was assessed by asking students to indicate how long they spend watching commercial television on a usual school day, Saturday and Sunday (recorded in hours and minutes). These responses were then used to calculate students' weighted average daily time spent watching commercial television.

Sleep duration was determined by computing the difference between students' usual wake-up time on a school day and bedtime on a school night.

Each variable measuring students' eating, physical activity, television viewing and sleeping patterns was binary coded to indicate poor behaviours (e.g. low intake ( $\leq 1$ daily serving) of vegetables and fruit, at least weekly consumption of fast foods, television viewing $>2 \mathrm{~h} / \mathrm{d}$ (consistent with national guidelines) ${ }^{(22)}$ ).

\section{Perceptions of soft drinks}

Students were asked to indicate their level of agreement (from $1=$ 'strongly disagree' to $5=$ 'strongly agree') with the following statements: (i) 'Soft drinks are usually available in my home'; (ii) 'Soft drinks are convenient to buy'; and (iii) 'Soft drink is good value for money'.
Responses were collapsed into those who agreed $v$. those who disagreed or neither agreed nor disagreed.

\section{Purchasing of soft drinks at school}

Students nominated what kind of drink they usually buy from the (i) school canteen and (ii) school vending machine, respectively. Response options were: 'I don't buy drinks from the canteen/vending machine'; '100\% fruit juice'; 'sport drink'; 'water'; 'soft drink'; 'diet soft drink'; 'milk (plain or flavoured)'; and 'iced tea'. An additional response option of 'Our school doesn't have a vending machine' was provided for the school vending machine question only. A binary variable was created to identify students who reported usually buying soft drinks when making a beverage purchase from the school canteen and/or school vending machine.

\section{Statistical analyses}

Data were analysed using the statistical software package Stata SE 14.1 and weighted by state, education sector, school year and sex to bring the sample obtained in line with the population distribution. The 'svy' prefix command in Stata was used to account for the weighting, clustering and stratification of the survey design. Three separate multivariable logistic regression models were conducted to examine the association between high consumption of soft drinks and: (i) demographic, weight status and behavioural factors; (ii) students' perceptions of soft drinks; and (iii) the purchasing of these drinks at school. Each multivariable model adjusted for school type (government, Catholic and independent) and school-level clustering. The second and third models also controlled for demographic characteristics and weight status. Given the large sample size, a more conservative significance level of $P<0.01$ was applied.

\section{Results}

A total of 196 secondary schools were surveyed nationally (school response rate $=21 \%$ ), with data collected from 11044 students in Years 8 to 11 (student response rate $=53 \%$ ). The present paper reports on data for 7835 students aged 12-17 years who recorded their soft drink consumption and for whom complete demographic information is available.

Of the 7835 students included in the final sample for analyses, $51 \%$ were male. There was a lower proportion of students in Year 11 (20\%) compared with Years 8, 9 (both $27 \%$ ) and 10 (26\%). About one-third of students (35\%) were classified as low SEP, 36\% as medium SEP and $28 \%$ as high SEP (compared with 34\% low, $41 \%$ medium and $25 \%$ high for the Australian population when using the same SEP categorisation criteria) ${ }^{(23)}$, while $58 \%$ of students resided in a metropolitan location. 
Thirty-one per cent of students indicated they had less than \$AU 10 weekly spending money, $40 \%$ had between \$AU 10 and \$AU 39, and 30\% had at least \$AU \$40 available to spend on themselves during a typical week. Just over one in five students (22\%) were categorised as overweight or obese.

Overall, $14 \%$ of students reported consuming four or more cups of soft drinks per week, classifying them as high consumers. Adjusted proportions and odds of being a high soft drink consumer associated with each demographic, weight status and health behaviour characteristic (i.e. model 1) are presented in Table 1. After adjustment for all covariates in the model, high consumption of soft drinks was found to be independently associated with two demographic factors: sex and weekly spending money. Males were more likely than were females to be high soft drink consumers, while students who had at least \$AU 40 per week to spend on themselves were more likely than those who had less than \$AU 10 available in weekly spending money to consume a high amount of soft drinks. The proportion of students who were high consumers of soft drinks did not vary significantly by weight status.

In terms of health behaviours, students who reported eating one serving or less of fruit were more likely to be high soft drink consumers than those who ate two or more daily servings of fruit. The odds of being a high soft drink consumer were also greater among students who reported eating fast foods at least once weekly and snack foods fourteen or more times per week (equivalent to two or more times daily on average), as well as those who consumed energy drinks on a weekly basis. While students' physical activity behaviour was unrelated to their consumption of soft drinks, there were significant associations found for both television viewing and sleep duration. Spending more than $2 \mathrm{~h}$ watching television daily and sleeping for less than $8 \mathrm{~h}$ on a usual school night were both independently associated with increased odds of consuming a high amount of soft drinks.

Table 2 presents the results from the second multivariable model examining the associations between students' perceptions of soft drinks and their consumption of these drinks. After controlling for demographic characteristics and weight status, the odds of being classified as a high soft drink consumer were about four times higher among students who agreed that soft drinks are usually available in their home compared with those who did not hold this perception. Believing that soft drinks are convenient to buy and are good value for money also significantly increased the odds of students being classified as high consumers, but to a lesser degree (only $\sim 1.5$ times higher).

Finally, the third multivariable model found that, after controlling for demographic characteristics and weight status, students who reported usually buying a soft drink when making a beverage purchase from the school canteen and/or school vending machine were significantly more likely to be a high soft drink consumer compared with students who either did not purchase drinks at school or more commonly bought other types of beverages at school (adjusted \%: 29.7 v. 11.6; adjusted OR =3.47; 95\% CI $2 \cdot 74,4 \cdot 39, P<0 \cdot 001)$.

\section{Discussion}

Overall, the results of the current study indicate that high consumption of soft drinks among Australian secondaryschool students is more prevalent among males and those with more weekly spending money. High soft drink consumption also clusters with a number of other unhealthy lifestyle behaviours, namely low intake of fruit, high intakes of fast foods and snack foods, weekly consumption of energy drinks, greater exposure to commercial television and short sleep duration. To our knowledge, ours is the first national study conducted in Australia to show that both dietary and sedentary behaviours are independently associated with high soft drink consumption within an adolescent population.

The finding that male students are more likely to be high consumers of soft drinks compared with female students is in line with earlier studies conducted both in Australia $^{(24)}$ and overseas ${ }^{(11-13)}$. While similar gender differences are evident across all age groups, consumption levels are at their highest during adolescence and young $\operatorname{adulthood}^{(7)}$, making this a critical period in which to intervene and implement strategies with a specific focus on reducing soft drink intake among males. Adolescents are at a stage in their life where they are beginning to exert greater control over their own dietary choices as they gain more independent buying power (e.g. through having a part-time job and earning their own money), which likely influences the types of drinks they consume. Indeed, we noted that students with higher amounts of available weekly spending money were more likely to be high soft drink consumers, suggesting at least some purchasing of soft drinks by adolescents themselves.

Students residing in low-SEP neighbourhoods were no more likely to be high soft drink consumers than those who lived in high-SEP neighbourhoods. Socio-economic disparities in adolescents' soft drink consumption have previously been reported in cross-national studies of school-aged children and adolescents in Europe, albeit with some variations between countries and genders, when using parental occupation, parental education and familial wealth as indicators of $\mathrm{SEP}^{(25-28)}$. In Australia, mean intake of soft drinks has been shown to be higher among 14-16-year-olds living in low-SEP areas compared with those residing in high-SEP areas; however, the difference between the highest and lowest SEP groups in the proportion consuming soft drinks on the day of the survey was not found to be statistically significant for this age group $^{(8)}$. For future studies, it would be beneficial to include individual-level indicators such as household 
Table 1 Results from a multivariable logistic regression analysis examining demographic and behavioural correlates of soft drink consumption among Australian secondary-school students $(n 7750)^{\star}$ aged $12-17$ years; 2012-13 National Secondary Students' Diet and Activity (NaSSDA) survey

\begin{tabular}{|c|c|c|c|c|}
\hline \multirow[b]{3}{*}{ Covariate } & \multicolumn{4}{|c|}{ Soft drink consumption ( $\geq 4$ cups/week) } \\
\hline & \multicolumn{4}{|c|}{ Multivariable model 1} \\
\hline & Adjusted \%† & Adjusted OR† & $95 \% \mathrm{Cl}$ & $P$ value \\
\hline \multicolumn{5}{|l|}{ Demographic characteristics } \\
\hline \multicolumn{5}{|l|}{ Sex } \\
\hline Male & $16 \cdot 8$ & $2 \cdot 11$ & $1.69,2.64$ & $<0.001$ \\
\hline Female & 9.6 & 1.00 & Ref. & - \\
\hline \multicolumn{5}{|l|}{ Year level } \\
\hline 8 & $13 \cdot 6$ & 1.00 & Ref. & - \\
\hline 9 & $14 \cdot 8$ & $1 \cdot 13$ & $0.85,1.49$ & 0.401 \\
\hline 10 & $13 \cdot 0$ & 0.94 & $0.72,1.23$ & 0.645 \\
\hline 11 & $13 \cdot 3$ & 0.97 & $0.73,1.28$ & 0.815 \\
\hline \multicolumn{5}{|l|}{ Socio-economic position } \\
\hline Low & 14.5 & 1.00 & Ref. & - \\
\hline Medium & 14.4 & 0.99 & $0.76,1.30$ & 0.946 \\
\hline High & $11 \cdot 0$ & 0.69 & $0.49,0.99$ & 0.044 \\
\hline \multicolumn{5}{|l|}{ Geographic location } \\
\hline Metropolitan & $14 \cdot 3$ & 1.00 & Ref. & - \\
\hline Rural/regional & $12 \cdot 5$ & 0.84 & $0.64,1.10$ & 0.193 \\
\hline \multicolumn{5}{|l|}{ Weekly spending money } \\
\hline$<\$ A U 10$ & $11 \cdot 6$ & 1.00 & Ref. & - \\
\hline \$AU 10-\$AU 39 & $13 \cdot 4$ & 1.22 & $0.92,1.60$ & 0.163 \\
\hline$\geq \$ A U 40$ & $15 \cdot 9$ & 1.53 & $1.20,1.96$ & 0.001 \\
\hline \multicolumn{5}{|l|}{ Weight status } \\
\hline \multicolumn{5}{|l|}{ BMI categorył } \\
\hline Healthy weight/underweight & $13 \cdot 6$ & 1.00 & Ref. & - \\
\hline Overweight/obese & $13 \cdot 9$ & 1.03 & $0.82,1.28$ & 0.812 \\
\hline \multicolumn{5}{|l|}{ Health behaviours } \\
\hline \multicolumn{5}{|l|}{ Vegetable consumption } \\
\hline$\leq 1$ serving/d & $14 \cdot 8$ & $1 \cdot 19$ & $0.96,1.47$ & 0.120 \\
\hline$\geq 2$ servings/d & $13 \cdot 1$ & 1.00 & Ref. & - \\
\hline \multicolumn{5}{|l|}{ Fruit consumption } \\
\hline$\leq 1$ serving/d & $17 \cdot 6$ & $1 \cdot 70$ & $1 \cdot 35,2 \cdot 15$ & $<0.001$ \\
\hline$\geq 2$ servings/d & 11.9 & 1.00 & Ref. & - \\
\hline \multicolumn{5}{|l|}{ Fruit juice consumption } \\
\hline$\leq 3$ cups/week & $13 \cdot 0$ & 1.00 & Ref. & - \\
\hline$\geq 4$ cups/week & $14 \cdot 8$ & $1 \cdot 19$ & $0.92,1.54$ & 0.183 \\
\hline \multicolumn{5}{|l|}{ Energy drink consumption } \\
\hline$<1 \mathrm{cup} /$ week & $12 \cdot 2$ & 1.00 & Ref. & - \\
\hline$\geq 1$ cup/week & $20 \cdot 3$ & 2.01 & $1.59,2.54$ & $<0.001$ \\
\hline \multicolumn{5}{|l|}{ Fast-food consumption } \\
\hline$<1$ time/week & $9 \cdot 3$ & 1.00 & Ref. & - \\
\hline$\geq 1$ times/week & $18 \cdot 1$ & $2 \cdot 36$ & $1 \cdot 98,2 \cdot 80$ & $<0.001$ \\
\hline \multicolumn{5}{|l|}{ Snack food consumption } \\
\hline$\leq 13$ times/week & $11 \cdot 3$ & 1.00 & Ref. & - \\
\hline \multirow{2}{*}{\multicolumn{5}{|c|}{ Physical activity }} \\
\hline & & & & \\
\hline$\leq 3 \mathrm{~d} /$ week & $13 \cdot 9$ & 1.05 & $0.85,1.29$ & 0.659 \\
\hline$\geq 4 \mathrm{~d} /$ week & 13.5 & 1.00 & Ref. & - \\
\hline Television viewing & & & & \\
\hline$\leq 2 \mathrm{~h} / \mathrm{d}$ & $12 \cdot 1$ & 1.00 & Ref. & - \\
\hline$>2 \mathrm{~h} / \mathrm{d}$ & $16 \cdot 2$ & 1.48 & $1 \cdot 21,1 \cdot 81$ & $<0.001$ \\
\hline Sleep & & & & \\
\hline$<8 \mathrm{~h} / \mathrm{school}$ night & $16 \cdot 0$ & 1.34 & $1.09,1.65$ & 0.005 \\
\hline$\geq 8 \mathrm{~h} / \mathrm{school}$ night & $12 \cdot 9$ & 1.00 & Ref. & - \\
\hline
\end{tabular}

Ref., referent category.

*Sample size smaller due to missing values.

†Adjusted for all other covariates listed in the table, school-level clustering and school type.

$\ddagger$ Łased on internationally recognised BMI cut-offs developed for children and adolescents ${ }^{(18)}$.

income to further elucidate the relationship between SEP and adolescents' soft drink consumption within the Australian context.
In our study, high soft drink consumption was unrelated to measured weight status, a result that concurs with findings from two national school-based surveys conducted 
Table 2 Associations between soft drink consumption and perceptions of soft drink availability, convenience and value for money among Australian secondary-school students ( $n$ 7835) aged $12-17$ years; 2012-13 National Secondary Students' Diet and Activity (NaSSDA) survey

\begin{tabular}{|c|c|c|c|c|}
\hline \multirow[b]{3}{*}{ Covariate } & \multicolumn{4}{|c|}{ Soft drink consumption ( $\geq 4$ cups/week) } \\
\hline & \multicolumn{4}{|c|}{ Multivariable model 2} \\
\hline & Adjusted \%* & Adjusted OR* ${ }^{*}$ & $95 \% \mathrm{Cl}$ & $P$ value \\
\hline \multicolumn{5}{|c|}{ Soft drinks are usually available in my home } \\
\hline Disagree/neither & $7 \cdot 4$ & 1.00 & Ref. & - \\
\hline Agree & $23 \cdot 2$ & 4.11 & $3.32,5.09$ & $<0.001$ \\
\hline \multicolumn{5}{|c|}{ Soft drinks are convenient to buy } \\
\hline Disagree/neither & 11.5 & 1.00 & Ref. & - \\
\hline Agree & $16 \cdot 1$ & 1.56 & $1.23,1.98$ & $<0.001$ \\
\hline \multicolumn{5}{|c|}{ Soft drink is good value for money } \\
\hline Disagree/neither & 12.5 & 1.00 & Ref. & - \\
\hline Agree & $17 \cdot 3$ & 1.55 & $1.22,1.98$ & $<0.001$ \\
\hline
\end{tabular}

Ref., referent category.

*Adjusted for all other covariates listed in the table, demographic characteristics, weight status, school-level clustering and school type.

in the USA ${ }^{(11,12)}$. However, it contrasts with a systematic review and meta-analysis of prospective cohort studies and randomised controlled trials that concluded there was evidence of a positive association between SSB consumption and body weight gain in children and adolescents ${ }^{(2)}$. It is possible that overweight students may have underreported their soft drink consumption in the present study. Alternatively, they may be consuming high quantities of other types of SSB such as cordial and fruit drinks. Overweight students may also have moderated their intake of soft drinks as a weight control method, an explanation that is supported by evidence that dieting ${ }^{(13)}$ and trying to lose weight $^{(12)}$ are associated with decreased frequency of consuming soft drinks among high-school students.

The observation that soft drink consumption clusters with other unhealthy dietary behaviours has been reported previously, with adolescent studies also finding intake of these drinks to be positively associated with consumption of fast foods ${ }^{(11,29,30)}$, snack foods (e.g. potato crisps, ice cream) $)^{(20,30)}$ and energy drinks ${ }^{(31)}$. Further, we found that students who reported low consumption of fruit, but not vegetables, had increased odds of being high soft drink consumers. This pattern corresponds with results from a 2007 national survey of Australian children that examined factors associated with high consumption of carbonated drinks (i.e. soft drinks and energy drinks) using $2 \mathrm{~d}$ dietary recall data ${ }^{(24)}$. Given the apparent clustering of unhealthy dietary practices among adolescents, it is crucial that interventions aimed at reducing intake of soft drinks monitor how changes in consumption of these types of beverages may possibly impact on other eating behaviours. In addition, whole-of-diet interventions that recognise these interrelationships may prove more effective than interventions with a singular focus on soft drinks.

While some previous US studies have found there to be an inverse association between students' physical activity behaviour and soft drink consumption ${ }^{(10,11)}$, no such evidence was forthcoming in the present study (or the 2007 Australian National Children's Nutrition and Physical Activity Survey) ${ }^{(8)}$. We used a relatively blunt physical activity measure, which combined moderate and vigorous activity into a single, self-reported question. However, factors such as the duration and intensity of the activity and the setting/behavioural context in which it occurs likely impact on adolescents' rehydration needs and the beverage choices available to them. For example, soft drinks have been identified as one of the top-selling food and drink items sold in canteens and vending machines at community-level children's sports clubs in two Australian States/Territories ${ }^{(32)}$. Thus, it is important that future studies aim to validate our null finding using multiple, and more specific, indicators of physical activity (e.g. time spent participating in organised sport outside school).

Higher television viewing ( $>2 \mathrm{~h} / \mathrm{d}$ ) was related to high soft drink consumption, in line with findings from a systematic review showing clear associations among adolescents between sedentary behaviour and unhealthy dietary practices, including intake of energy-dense drinks ${ }^{(33)}$. The effects of television viewing on diet have commonly been attributed to food advertising; however, non-advertising mechanisms, such as distraction and interruption of physiological food regulation (e.g. satiety cues), may also possibly explain this link ${ }^{(34)}$. As observed in the 2009 US Youth Risk Behavior Survey ${ }^{(12)}$, we found that students who reported shorter sleep duration ( $<8 \mathrm{~h} /$ school night) were more likely to be high soft drink consumers. Caffeine is a common additive used in cola soft drinks to enhance flavour ${ }^{(35)}$ and high caffeine intake among adolescents (primarily in the form of soft drinks) is correlated with difficulty sleeping and feeling tired in the morning ${ }^{(36)}$. The removal of caffeine (which reduces the sweetness of sucrose) from soft drinks could have potential benefits beyond improving sleep quality, by 
allowing for a reduction in sucrose (and by extension energy content) without affecting flavour ${ }^{(37)}$.

Consistent with previous research ${ }^{(13,38,39)}$, we found evidence of a strong link between the perceived availability of soft drinks in the home and students' consumption of these drinks. With the majority of Australian adolescents' intake of SSB (which includes soft drinks) occurring at home ${ }^{(24)}$, it is important that parents and children are better educated about the negative health impacts of soft drinks. One possible population-level educational strategy that warrants careful consideration is the introduction of mandatory health warning labels on soft drinks. Health warning labels on tobacco packages and alcohol containers have been shown to have positive effects on knowledge, attitudes, intentions and/or behaviour $^{(40-44)}$; however, their utility on soft drinks is less clear. Recent experimental study findings indicate that SSB health warning labels are able to reduce adolescents' and young people's preferences for SSB in hypothetical purchasing scenarios ${ }^{(45,46)}$. Further research is needed to assess how adolescents respond to such warning labels on soft drinks, rather than SSB more broadly.

Students who perceived that soft drinks are convenient to buy and good value for money were more likely to be consuming a high amount of soft drinks, reflecting a need for interventions that reduce the accessibility of these drinks. Increasing the price of soft drinks via a tax on SSB could have potential to discourage consumption and influence demand among adolescents for healthier alternatives such as water. For example, in Mexico, where a tax on SSB was introduced at the beginning of 2014, a small decline has been observed in the average volume of household SSB purchases and an increase in purchases of untaxed beverages (in particular bottled water) ${ }^{(47)}$. In addition, economic studies suggest that consumers are sensitive to price changes for soft drinks, with higher prices corresponding to lower consumption ${ }^{(48-50)}$. Within the Australian context, it is estimated that an excise tax of 40 cents per $100 \mathrm{~g}$ of sugar contained within SSB would reduce consumption by approximately $15 \%$ and generate about \$AU 500 million in annual revenue that could be used to fund obesity prevention initiatives ${ }^{(51)}$.

In our study, students had significantly greater odds of being a high soft drink consumer if they usually bought soft drinks when making a beverage purchase at school. This finding indicates that the availability of soft drinks for purchase by students in school settings may be facilitating their high consumption. While the percentage of SSB consumed at school by Australian adolescents is much lower than at home $(\sim 11 v . \sim 63 \%)^{(24)}$, this percentage could be substantially reduced through implementation of a total ban on soft drinks in schools. At present, Australian State Government policy guidelines generally impose restrictions on the sale of these drinks in school canteens. Yet, many secondary schools do not adhere to these guidelines, with an overall lack of monitoring and enforcement likely contributing to non-compliance ${ }^{(52)}$. Thus, any future legislative attempts to prohibit soft drinks from being sold in schools should be supported by a stringent monitoring and enforcement system to increase compliance.

Some study limitations should be acknowledged. First, given the cross-sectional study design, we are unable to draw any causal inferences regarding the observed associations. Second, due to survey length constraints, we used a single item question with response categories to measure soft drink consumption rather than a more rigorous dietary assessment tool (e.g. FFQ, 24 h dietary recall). Third, the data were self-reported and may be affected by recall and social desirability. For example, prior research suggests that young people have a tendency to underestimate their intake of soft drinks when responding to a short dietary question ${ }^{(53)}$. Fourth, we did not examine correlates of other types of SSB such as energy drinks, fruit drinks, sports drinks and cordial. As such, our findings are specific to soft drinks and cannot be generalised to all SSB. Finally, the response rates achieved were relatively low, reflecting the growing challenges researchers face in gaining access to schools and recruiting students using active consent procedures. However, the use of replacement schools with similar characteristics to originally selected schools did, to some extent, preserve the representativeness of the large sample.

\section{Conclusion}

The results of the current large, national, school-based study highlight the clustering of high soft drink consumption with other unhealthy lifestyle behaviours among Australian secondary-school students. Interventions that strive to reduce the availability of soft drinks (e.g. increased taxes, restricting their sale in schools), as well as improved population-level education on the harms of these drinks, are needed to lower adolescents' soft drink intake.

\section{Acknowledgements}

Acknowledgements: The NaSSDA Study Team comprises Cancer Council Victoria: Belinda Morley, Maree Scully, Philippa Niven, Melanie Wakefield; Technical Advisory Group: Louise Baur (Chair), David Crawford, Victoria Flood, Anthony Okely, Iain S. Pratt, Jo Salmon. The authors thank the school principals, teachers and students who participated in the study, and I-view who were responsible for fieldwork coordination. Financial support: The NaSSDA survey was jointly funded by State Cancer Councils through Cancer Council Australia, the National Heart Foundation of Australia, and the State and Territory Government Health Departments. M.W. is supported by a Principal Research Fellowship from the National Health and Medical Research Council. The funders had no role in the design, analysis or writing of this article. Conflict of interest: None. Authorship: All authors have contributed to the present paper by being 
involved in conceiving and designing the study or in analysis and interpretation of the data, and in writing and revising the paper. Ethics of human subject participation: The NaSSDA Study was approved by the Human Research Ethics Committee of Cancer Council Victoria. In addition, approval was obtained from the education authority for each sector in each State and Territory, as well as the principal at each selected school.

\section{References}

1. Vartanian LR, Schwartz MB \& Brownell KD (2007) Effects of soft drink consumption on nutrition and health: a systematic review and meta-analysis. Am J Public Health 97, 667-675.

2. Malik VS, Pan A, Willett WC et al. (2013) Sugar-sweetened beverages and weight gain in children and adults: a systematic review and meta-analysis. Am J Clin Nutr 98 , 1084-1102.

3. Te Morenga L, Mallard S \& Mann J (2012) Dietary sugars and body weight: systematic review and meta-analyses of randomised controlled trials and cohort studies. BMJ $\mathbf{3 4 6}$, e7492.

4. World Health Organization (2015) Guideline: Sugars Intake for Adults and Children. Geneva: WHO.

5. Moynihan P \& Petersen PE (2004) Diet, nutrition and the prevention of dental diseases. Public Health Nutr $\mathbf{7}$, 201-226.

6. Shenkin JD, Heller KE, Warren JJ et al. (2003) Soft drink consumption and caries risk in children and adolescents. Gen Dent 51, 30-36.

7. Australian Bureau of Statistics (2014) Non-Alcoholic Beverages. 4364.0.55.007 - Australian Health Survey: Nutrition First Results - Foods and Nutrients, 2011-12. Canberra: ABS

8. Clifton PM, Chan L, Moss CL et al. (2011) Beverage intake and obesity in Australian children. Nutr Metab (Lond) 8, 87.

9. Rangan AM, Kwan J, Flood VM et al. (2011) Changes in 'extra' food intake among Australian children between 1995 and 2007. Obes Res Clin Pract 5, e55-e63.

10. Ranjit N, Evans MH, Byrd-Williams C et al. (2010) Dietary and activity correlates of sugar-sweetened beverage consumption among adolescents. Pediatrics 126, e754-e761.

11. Park S, Blanck HM, Sherry B et al. (2012) Factors associated with sugar-sweetened beverage intake among United States high school students. J Nutr 142, 306-312.

12. Park S, Sherry B, Foti K et al. (2012) Self-reported academic grades and other correlates of sugar-sweetened soda intake among US adolescents. I Acad Nutr Diet 112, 125-131.

13. Bere E, Glomnes ES, te Velde SJ et al. (2008) Determinants of adolescents' soft drink consumption. Public Health Nutr 11, 49-56.

14. Stokols D (1992) Establishing and maintaining healthy environments. Toward a social ecology of health promotion. Am Psychol 47, 6-22.

15. Australian Bureau of Statistics (2013) Technical Paper: Socio-Economic Indexes for Areas (SEIFA) 2011. ABS Catalogue no. 2033.0.55.001. Canberra: ABS.

16. Australian Bureau of Statistics (2013) Australian Statistical Geography Standard (ASGS): Volume 5 - Remoteness Structure. ABS Catalogue no. 1270.0.55.005. Canberra: ABS.

17. Davies PSW, Roodveldt R \& Marks G (2001) Standard Methods for the Collection and Collation of Anthropometric Data in Children. Canberra: Commonwealth Department of Health and Aged Care.

18. Cole TJ, Bellizzi MC, Flegal KM et al. (2000) Establishing a standard definition for child overweight and obesity worldwide: international survey. BMJ 320, 1240-1243.
19. Flood V, Webb K \& Rangan A (2005) Recommendations for Short Questions to Assess Food Consumption in Children for the NSW Health Surveys. Sydney: NSW Centre for Public Health Nutrition.

20. Niven P, Scully M, Morley B et al. (2015) What factors are associated with frequent unhealthy snack-food consumption among Australian secondary-school students? Public Health Nutr 18, 2153-2160.

21. Prochaska JJ, Sallis JF \& Long B (2001) A physical activity screening measure for use with adolescents in primary care. Arch Pediatr Adolesc Med 155, 554-559.

22. Department of Health (2014) Australia's Physical Activity and Sedentary Behaviour Guidelines. Canberra: Commonwealth of Australia.

23. Australian Bureau of Statistics (2013) 2033.0.55.001 Census of Population and Housing: Socio-Economic Indexes for Areas (SEIFA), Australia, 2011. Canberra: ABS.

24. Hafekost K, Mitrou F, Lawrence D et al. (2011) Sugar sweetened beverage consumption by Australian children: implications for public health strategy. BMC Public Health 11, 950 .

25. Vereecken CA, Inchley J, Subramanian SV et al. (2005) The relative influence of individual and contextual socio-economic status on consumption of fruit and soft drinks among adolescents in Europe. Eur J Public Health 15, 224-232.

26. Brug J, van Stralen MM, Te Velde SJ et al. (2012) Differences in weight status and energy-balance related behaviors among schoolchildren across Europe: the ENERGY-project. PLoS One 7, e34742.

27. Currie C, Molcho M, Boyce W et al. (2008) Researching health inequalities in adolescents: the development of the Health Behaviour in School-Aged Children (HBSC) Family Affluence Scale. Soc Sci Med 66, 1429-1436.

28. Inchley J, Currie D, Young $\mathrm{T}$ et al. (2016) Growing up Unequal: Gender and Socioeconomic Differences in Young People's Health and Well-Being. Copenhangen: WHO Regional Office for Europe.

29. Verzeletti C, Maes L, Santinello M et al. (2010) Soft drink consumption in adolescence: associations with food-related lifestyles and family rules in Belgium Flanders and the Veneto Region of Italy. Eur J Public Health 20, 312-317.

30. Collison KS, Zaidi MZ, Subhani SN et al. (2010) Sugarsweetened carbonated beverage consumption correlates with BMI, waist circumference, and poor dietary choices in school children. BMC Public Health 10, 234.

31. Larson N, Dewolfe J, Story M et al. (2014) Adolescent consumption of sports and energy drinks: linkages to higher physical activity, unhealthy beverage patterns, cigarette smoking, and screen media use. J Nutr Educ Behav 46, 181-187.

32. Kelly B, Baur LA, Bauman AE et al. (2010) Examining opportunities for promotion of healthy eating at children's sports clubs. Aust N Z J Public Health 34, 583-588.

33. Pearson N \& Biddle SJH (2011) Sedentary behavior and dietary intake in children, adolescents, and adults. A systematic review. Am J Prev Med 41, 178-188.

34. Marsh S, Ni Mhurchu C \& Maddison R (2013) The nonadvertising effects of screen-based sedentary activities on acute eating behaviours in children, adolescents, and young adults. A systematic review. Appetite 71, 259-273.

35. Keast RS \& Riddell LJ (2007) Caffeine as a flavor additive in soft-drinks. Appetite 49, 255-259.

36. Orbeta RL, Overpeck MD, Ramcharran D et al. (2006) High caffeine intake in adolescents: associations with difficulty sleeping and feeling tired in the morning. $J$ Adolesc Health 38, 451-453.

37. Keast RS, Sayompark D, Sacks G et al. (2011) The influence of caffeine on energy content of sugar-sweetened beverages: 'the caffeine-calorie effect'. Eur J Clin Nutr 65, 1338-1344. 
38. Denney-Wilson E, Crawford D, Dobbins T et al. (2009) Influences on consumption of soft drinks and fast foods in adolescents. Asia Pac J Clin Nutr 18, 447-452.

39. Grimm GC, Harnack L \& Story M (2004) Factors associated with soft drink consumption in school-aged children. $J$ Am Diet Assoc 104, 1244-1249.

40. Hammond D (2011) Health warning messages on tobacco products: a review. Tob Control 20, 327-337.

41. Martin-Moreno JM, Harris ME, Breda J et al. (2013) Enhanced labelling on alcoholic drinks: reviewing the evidence to guide alcohol policy. Eur J Public Health 23, 1082-1087.

42. Pettigrew S, Jongenelis MI, Glance D et al. (2016) The effect of cancer warning statements on alcohol consumption intentions. Health Educ Res 31, 60-69.

43. Azagba S \& Sharaf MF (2013) The effect of graphic cigarette warning labels on smoking behavior: evidence from the Canadian experience. Nicotine Tob Res 15, 708-717.

44. White V, Webster B \& Wakefield M (2008) Do graphic health warning labels have an impact on adolescents' smokingrelated beliefs and behaviors? Addiction 103, 1562-1571.

45. VanEpps EM \& Roberto CA (2016) The influence of sugarsweetened beverage warnings: a randomized trial of adolescents' choices and beliefs. Am J Prev Med 51, 664-672.

46. Bollard T, Maubach N, Walker N et al. (2016) Effects of plain packaging, warning labels, and taxes on young people's predicted sugar-sweetened beverage preferences: an experimental study. Int J Behav Nutr Phys Act 13, 95.
47. Colchero MA, Popkin BM, Rivera JA et al. (2016) Beverage purchases from stores in Mexico under the excise tax on sugar sweetened beverages: observational study. BMJ 352, h6704.

48. Andreyeva T, Chaloupka FJ \& Brownell KD (2011) Estimating the potential of taxes on sugar-sweetened beverages to reduce consumption and generate revenue. Prev Med $\mathbf{5 2}$, 413-416.

49. Andreyeva T, Long MW \& Brownell KD (2010) The impact of food prices on consumption: a systematic review of research on the price elasticity of demand for food. Am J Public Health 100, 216-222.

50. Thow AM, Downs S \& Jan S (2014) A systematic review of the effectiveness of food taxes and subsidies to improve diets: understanding the recent evidence. Nutr Rev 72, 551-565.

51. Duckett S, Swerissen H \& Wiltshire T (2016) A Sugary Drinks Tax: Recovering the Community Costs of Obesity. Melbourne: Grattan Institute; available at http:/grattan. edu.au/wp-content/uploads/2016/11/880-A-sugary-drinkstax.pdf

52. Woods J, Bressan A, Langelaan C et al. (2014) Australian school canteens: menu guideline adherence or avoidance? Health Promot J Aust 25, 110-115.

53. Gwynn JD, Flood VM, D'Este CA et al. (2011) The reliability and validity of a short FFQ among Australian Aboriginal and Torres Strait Islander and non-Indigenous rural children. Public Health Nutr 14, 388-401. 